\title{
HOMOLOGICALLY HOMOGENEOUS RINGS
}

\author{
BY
}

\author{
K. A. BROWN AND C. R. HAJARNAVIS
}

\begin{abstract}
In this paper we study the structure of a right Noetherian ring $R$ of finite right global dimesion integral over a central subring $C$ and satisfying the following condition:

if $V, W$ are irreducible right $R$-modules with $r_{C}(V)=r_{C}(W)$ then $\operatorname{pr} \operatorname{dim}(V)=$ $\operatorname{pr} \operatorname{dim}(W)$.
\end{abstract}

1. Introduction. Let $R$ be a ring with a central subring $C$. We shall say that $R$ is homologically homogeneous (hom. hom.) over $C$ if (i) $R$ is right Noetherian; (ii) $R$ is integral over $C$; (iii) the right global (projective) dimension of $R$ (denoted rt $g l \operatorname{dim}(R)$ ) is finite; and (iv) if $V$ and $M$ are irreducible right $R$-modules whose annihilators in $C$, namely $r_{C}(V)$ and $r_{C}(W)$, are equal then $\operatorname{pr} \operatorname{dim}_{R}(V)=$ $\operatorname{pr} \operatorname{dim}_{R}(W)$. (Here, $\operatorname{pr} \operatorname{dim}_{R}(V)$ denotes the projective dimension of the $R$-module $V$.) This paper is devoted to the study of such rings. Examples of them include (a) commutative Noetherian rings of finite global dimension; (b) hereditary Noetherian rings integral over their centers with no Artinian ideals; (c) right Noetherian local rings of finite right global dimension which are integral over their centers; and (d) certain rings arising in the representation theory of semisimple Lie groups [2]. In addition, we show in $\$ 7$ that polynomial rings over hom. hom. rings, and certain group rings, are hom. hom.

Rings of type (c) were studied in [5] where we showed that their maximal ideals had rank equal to their grade, defined in terms of maximal lengths of $C$-sequences. We termed such rings $C$-Macaulay-a precise definition is given in $\$ 2-$ and showed in $[5, \S 4]$ that they share many of the properties of commutative Cohen-Macaulay rings. The key to the study of a ring $R$ hom. hom. over $C$ is Theorem $2.5-$ if $R$ is hom. hom over $C$, then it is $C$-Macaulay. We list now the main results of this paper, which all flow more or less directly from this fact.

(i) $\mathrm{cl} k-\operatorname{dim}(R)=k-\operatorname{dim}(R)=\mathrm{rtgl} \operatorname{dim}(R)$, (where $\mathrm{cl} k$-dim( ) [resp. $k$-dim( )] denote the classical [resp. Gabriel-Rentschler [9]] Krull dimensions), (2.5).

(ii) For all prime ideals $P$ of $R$, the rank of $P$ equals its grade (2.6).

(iii) $R$ and $C$ satisfy the saturated chain condition on prime ideals, (2.6).

(iv) $R$ is hom. hom. over $C$ if and only if it is hom. hom. over its center $Z$, (2.7). (So the reference to $C$ in the definition of a hom. hom. ring is superfluous.)

(v) The localization of $R$ at a semiprime ideal of $C$ is again hom. hom., (3.5); this fact is used crucially in the proofs of the remaining properties.

Received by the editors December 20, 1982.

1980 Mathematics Subject Classification. Primary 16A60, 16A33.

(C)1984 American Mathematical Society $0002-9947 / 84 \$ 1.00+\$ .25$ per page 
(vi) $R$ is a finite direct sum of prime hom. hom. rings, (5.3).

(vii) The center of $R$ is a finite direct sum of Krull domains (6.1).

(viii) If $\left\{x_{1}, \ldots, x_{t}\right\}$ is a $C$-sequence on $R$, then $R / \Sigma_{i} x_{i} R$ has a quasi-Frobenius quotient ring, (4.4).

For rings of types (a) and (b) these results are of course well known (and do not require the integrality of $R$ over $C$ in case (b)). For rings of type (c) some of the results were obained in [5], to which this paper is a sequel, and for a ring finitely generated over its semilocal center versions of (i), (ii) and (iii) were obtained in [21]. A question of $\mathrm{J}$. Bernstein concerning rings of type (d) is answered by (i). We are grateful to Professors J. Bernstein and M. Artin for bringing this question to our attention. We would also like to thank Professor Artin for several helpful conversations on these matters and in particular for sketching to us an outline proof of (vi).

Some further results and open questions are touched on in $§ 8$.

All rings are assumed to contain an identity; all modules are unital, and are right modules unless otherwise described. We shall follow the notation of [5] and we refer the reader to that paper for some of the elementary properties.

The research described here was done while the first author was attending the 1981-82 Ring Theory Symposium at the University of Warwick. He thanks the staff of the University of Warwick Mathematics Institute for their hospitality, and the United Kingdom S.E.R.C. for its financial support.

2. Centrally Macaulay rings. The main result of this section is Theorem 2.5.

2.1. Lemma. Let the right Noetherian ring $R$ be integral over the subring $C$. Let $\mathrm{m}$ be a maximal ideal of $C$, and $V$ a finitely generated $R$-module with $V_{\mathrm{m}}=0$. Then $\operatorname{pr} \operatorname{dim}_{R}(V)=\operatorname{pr} \operatorname{dim}_{R_{\mathrm{m}}}\left(V_{\mathrm{m}}\right)$.

Proof. Let $n \geqslant 0$, and let $W$ be an $R$-module with

$$
0 \neq E=\operatorname{Ext}_{R}^{n}(V, W) \text {. }
$$

Since $V$ is right Noetherian, $E_{\mathrm{m}}=\operatorname{Ext}_{R_{\mathrm{m}}}^{n}\left(V_{\mathrm{m}}, W_{\mathrm{m}}\right)$ as in [16, 11.58]. Since $E_{\mathrm{m}}=0$ we have $0 \neq E_{\mathrm{m}}=\operatorname{Ext}_{R_{\mathrm{m}}}^{n}\left(V_{m}, W_{m}\right)$. Hence, $\operatorname{pr} \operatorname{dim}_{R_{\mathrm{m}}}\left(V_{\mathrm{m}}\right) \geqslant \operatorname{pr} \operatorname{dim}_{R}(V)$. The reverse inequality follows from the exactness of localization.

2.2 COROllary. Let $R$ be a ring which is homologically homogeneous over a central subring $C$, and let $\mathrm{m}$ be a maximal ideal of $C$. Then $R_{\mathrm{m}}$ is homologically homogeneous over $C_{\mathrm{m}}$.

2.3. Lemma. (i) Let $R$ be a right Noetherian ring. Suppose that either $R$ is semilocal and $J(R)$ has the right $A R$ property or that $R$ is left Noetherian. If $R$ has finite right global dimension, then $\mathrm{rt} \mathrm{gl} \operatorname{dim}(R)=\sup \left\{\operatorname{pr} \operatorname{dim}_{R}(V): V\right.$ simple $\}$.

(ii) Let $R$ be hom. hom. of dimension $n$ over the local central subring $C$. Let $M$ be a finitely generated $R$-module with nonzero socle. Then $\operatorname{pr} \operatorname{dim}(M)=n$.

Proof. (i) [3, Proposition 1.1 and 4, 4.8].

(ii) [12, Theorem B, p. 124].

2.4. Let $C$ be a central subring of the right Noetherian ring $R$. If $I$ is an ideal of $R$ (or $C$ ) and $A$ is a finitely generated $R$-module, the length of a maximal $C$-sequence 
in $I$ on $A$ is called the $C$-grade of $I$ on $A$, denoted $G_{C}(I, A)$. (A $C$-sequence in $I$ on $A$ is an ordered sequence $\left\{x_{1}, \ldots, x_{n}\right\}$ of elements of $I \cap C$ such that $A\left(\sum_{i} x_{i} R\right) \neq A$ and each $x_{i+1}$ is a nonzero divisor on the module $A / A\left(\sum_{j=1}^{i} x_{j} R\right)$.) Further details may be found in $[5, \S 4]$, where it is shown that $G_{C}(I, A)$ is well defined. We write $G(I)$ for $G_{C}(I, R)$.

The ring $R$ is centrally Macaulay over $C$, or C-Macaulay, if $G_{C}(M, R)=\operatorname{rank}(M)$ for all maximal ideals $M$ of $R$. (The $\operatorname{rank}, \operatorname{rank}(P)$, of a prime ideal $P$ is the greatest integer $t$ for which there exists a chain $P=P_{0} \supsetneqq P_{1} \supsetneqq \ldots \supsetneqq P_{t}$ of prime ideals $P_{i}$ )

2.5. THEOREM. Let $R$ be a ring which is homologically homogeneous over the central subring $C$. Let $M$ be a maximal ideal of $R$. Then

$$
G_{C}(M)=\operatorname{rank}(M)=\operatorname{pr} \operatorname{dim}_{R}(R / M) .
$$

In particular, $R$ is $C$-Macaulay and $k-\operatorname{dim}(R)=\mathrm{rtgl} \operatorname{dim}(R)$.

Proof. Let $M \cap C=\mathrm{m}$, a maximal ideal of $C$. By [5, 4.7] and Lemma 2.1, $R$ can be replaced by $R_{\mathrm{m}}$; that is, we may assume that $C$ is local. By [5, 5.1 and 6.1],

$$
G_{C}(M) \leqslant \operatorname{rank}(M) \leqslant \operatorname{pr} \operatorname{dim}_{R}(R / M) ;
$$

these inequalities do not depend on the homological homogeneity of $R$. Let $x_{1}, \ldots, x_{l}$ be a maximal $C$-sequence in $\mathrm{m}$ on $R$, and let $I=\sum_{i} x_{i} R$. By $[5,3.4], R / I$ has nonzero socle. Now by $[7,2.1] J(R)$ has the AR property.

So

$$
\operatorname{pr} \operatorname{dim}_{R}(R / I)=\mathrm{rtgl} \operatorname{dim}(R)=\operatorname{pr} \operatorname{dim}(R / M)
$$

by 2.3 . However, $\operatorname{pr} \operatorname{dim}_{R}(R / I)=l$ by $[4,4.11]$. Hence

$$
G_{C}(M)=\operatorname{prdim}_{R}(R / M),
$$

as required. Since $k-\operatorname{dim}(R)=\sup \{\operatorname{rank} M: M$ a maximal ideal of $R\}[7,1.7]$, the last claim follows also.

2.6. Theorem 2.5 generalizes $[5,6.2]$ in which $R$ is assumed to be local, and [12, Theorem 170], in which $R$ is commutative. In view of 2.5 , all the results of $[5, \S \S 4$ and 5] apply to right Noetherian hom. hom. rings. In particular,

(a) for all primes ideals $P$ of $R, G_{C}(P)=\operatorname{rank}(P)[5,4.11]$;

(b) $R$ and $C$ satisfy the saturated chain condition on prime ideals [5, 5.2].

2.7. It follows easily from 2.5 that the apparent dependence, in the definition of a homologically homogeneous ring, on the central subring $C$ in question is illusory:

Proposition. Let $R$ be right Noetherian with center $Z$. Let $C$ be a subring of $Z$ over which $R$ is integral. Then $R$ is hom. hom. over $Z$ if and only if $R$ is hom. hom. over $C$.

Proof. Clearly, if $R$ is hom. hom. over $C$ then it is hom. hom over $Z$. Conversely, suppose $R$ is hom. hom. over $Z$. Let $M$ and $N$ be two maximal ideals of $R$ with $M \cap C=N \cap C$. Let $\mathfrak{m}=M \cap Z$. Now $\operatorname{rank}(\mathfrak{m})=\operatorname{rank}(M)$ is finite, by 2.5 . Hence $\operatorname{rank}(\mathrm{m})=\operatorname{rank}(M \cap C)$, since $Z$ is integral over $C$. Similarly, $\operatorname{rank}(N)=$ $\operatorname{rank}(N \cap Z)=\operatorname{rank}(N \cap C)$. Thus, $\operatorname{rank}(M)=\operatorname{rank}(N)$, so $\operatorname{prdim} \operatorname{dim}_{R}(R / M)=$ pr $\operatorname{dim}_{R}(R / N)$ by 2.5. That is, $R$ is hom. hom. over $C$. 
In view of this result, we shall sometimes not mention the central subring $C$ in question when describing a ring as homologically homogeneous.

3. Localization and homological grade. Our principal target in this section is Theorem 3.5 which generalizes Corollary 2.2 to localization at an arbitrary semiprime ideal of the center. As a by-product of its proof we obtain in 3.6 a homological characterization of the grade of an ideal.

3.1. Let $I$ be an ideal of $R$. The homological grade of $I$, denoted $\operatorname{gr}(I)$, is defined as

$$
\operatorname{gr}(I)=\inf \left\{n: \operatorname{Ext}_{R}^{n}(R / I, R) \neq 0\right\} .
$$

Let. $\operatorname{pr} \operatorname{dim}(R / I)=t<\infty$. Then clearly $0 \leqslant \operatorname{gr}(I) \leqslant t$. In general $\operatorname{gr}(I) \geqslant G(I)$; this is an immediate consequence of the following result, proved exactly as in the case where $R$ is commutative [12, Appendix 3.1].

THEOREM. Let $R$ be a ring with central subring $C$, and let $A$ and $B$ be $R$-modules. Let $x_{1}, \ldots, x_{n}$ be a $C$-sequence on $A$, with $B\left(\Sigma_{i} x_{i} R\right)=0$. Then

$$
\operatorname{Ext}_{R}^{n}(B, A) \cong \operatorname{Hom}_{R}\left(B, A / A\left(\sum_{i} x_{i} R\right)\right) .
$$

COROLlaRY. Let $R$ be a ring with central subring $C, I$ an ideal of $R$ and $x_{1}, \ldots, x_{n}$ a $C$-sequence in $I$ on $R$. Then $\operatorname{Ext}^{i}(R / I, R)=0$ for $i=0,1, \ldots, n-1$.

3.2. Lemma. Let $R$ be hom. hom. of dimension $n$ over the local central subring $C$. Let $P$ be a prime ideal of $R$ with $k-\operatorname{dim}(R / P)=1$. Then $\operatorname{pr} \operatorname{dim}_{R}(R / P)=n-1$.

Proof. Let $c \in \mathcal{E}(P)$. There is an exact sequence of right $R$-modules

$$
0 \rightarrow R / P \stackrel{\psi}{\rightarrow} R / P \rightarrow X \rightarrow 0
$$

where $\psi$ is the left multiplication by $c$ and $X$ is Artinian. By [12, Theorem $\mathrm{B}(4), \mathrm{p}$. 124] and Lemma 2.3, $\operatorname{prdim}_{R}(R / P) \geqslant n-1$. By [4,4.8], $\operatorname{pr}_{R} \operatorname{dim}_{R}(R / P) \leqslant n-1$.

3.3. An easy induction on the rank, just as in the commutative case [12, Theorem 138] using [5, 4.10], yields

Lemma. Let $C$ be a central subring of the right Noetherian ring $R, P$ a prime ideal of $R$. Then $G_{C}(P) \leqslant \operatorname{rank}(P)$.

3.4. Lemma. Let $R$ be a right Noetherian C-Macaulay ring, and let $\mathcal{S}$ be a multiplicatively closed subset of $C \backslash\{0\}$.

(i) If $P$ is a prime ideal of $R$ with $P \cap \delta=\varnothing$, then $G_{C}(P)=G_{C_{\S}}\left(P_{\S}\right)$.

(ii) $R_{5}$ is a $C_{\S}$-Macaulay ring.

Proof. By $\left[5,4.5\right.$ and 4.6], $G_{C}(P) \leqslant G_{C_{\S}}\left(P_{\S}\right)$. Hence

$$
\begin{aligned}
G_{C}(P) & \leqslant G_{C_{s}}\left(P_{\S}\right) \leqslant \operatorname{rank}\left(P_{\varsigma}\right), \quad \text { by } 3.3 \\
& \leqslant \operatorname{rank}(P)=G_{C}(P), \quad \text { by }[\mathbf{5}, 4.12] .
\end{aligned}
$$

Thus, equality holds throughout; (i) and (ii) are immediate. 
3.5. THEOREM. Let $R$ be a ring homologically homogeneous over the central subring $C$, and let $\mathfrak{p}$ be a semiprime ideal of $C$. Let $\mathrm{a}_{1}, \ldots, a_{t}$ be the primes of $C$ minimal over p. Then $R_{\mathfrak{p}}$ is hom. hom. over $C_{\mathfrak{p}}$, and

$$
\mathrm{rtgl} \operatorname{dim}\left(R_{\mathrm{p}}\right)=k-\operatorname{dim}\left(R_{\mathrm{p}}\right)=\max _{i}\left\{G_{C}\left(\mathfrak{a}_{i}\right)=\max _{i} \operatorname{rank}\left(\mathrm{a}_{i}\right)\right\} .
$$

Proof. Suppose that for $i=1,2, \ldots, t ; R_{\mathrm{a}_{i}}$ is hom. hom. over $C_{\mathrm{a}_{i}}$. By 2.5 and 2.3;

$$
\mathrm{rtgl} \operatorname{dim}\left(R_{\mathrm{a}_{i}}\right)=k-\operatorname{dim}\left(R_{\mathrm{a}_{i}}\right)=G_{C}\left(\mathrm{a}_{i}\right)=\operatorname{rank}\left(\mathrm{a}_{i}\right)
$$

for each $i$. Since $R_{\mathfrak{a}_{i}}$ is a localization of $R_{\mathfrak{p}}$ at a maximal ideal of $C_{\mathfrak{p}}, 2.1$ and 2.3 imply that $R_{\mathfrak{p}}$ is hom. hom. over $C_{\mathfrak{p}}$ and that

$$
\mathrm{rtgl} \operatorname{dim}\left(R_{\mathrm{p}}\right)=\max _{i}\left\{\mathrm{rtgl} \operatorname{dim}\left(R_{\mathrm{a}_{i}}\right)\right\} .
$$

Since clearly $k-\operatorname{dim}\left(R_{\mathfrak{p}}\right)=\max \left(k-\operatorname{dim}\left(R_{\mathrm{q}_{1}}\right)\right)$ we obtain all the equalities (1). It thus suffices to consider the case when $\mathfrak{p}$ is prime. Let

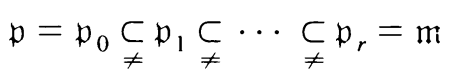

be a saturated chain of prime ideals of $C$ lying between $\mathfrak{p}$ and a maximal ideal $m$ of $C$. The proof is by induction on $r$; the case $r=0$ follows from 2.1, 2.5 and 3.4. Lemma 3.4 also shows that it suffices to treat the case $r=1$, which we now do; 3.4 and 2.2 show that we may assume that $C=C_{\mathrm{m}}$ is local.

Let $n=\operatorname{rank}(\mathrm{m})=G_{C}(\mathrm{~m})$. Then by $[5,5.2$ and 4.1]

$$
\operatorname{rank}(\mathfrak{p})=G_{C}(\mathfrak{p})=n-1 .
$$

Let $P$ be a prime ideal of $R$ with $P \cap C=\mathfrak{p}$, so that $k-\operatorname{dim}(R / P)=1$. By 3.2,

$$
\text { pr } \operatorname{dim}_{R}(R / P)=n-1 \text {. }
$$

By (2) and Corollary 3.1, $\operatorname{Ext}_{R}^{i}(R / P, R)=0$ for $0 \leqslant i \leqslant n-2$. Hence, by (3),

$$
\operatorname{Ext}_{R}^{i}(R / P, R) \neq 0 \Leftrightarrow i=n-1 .
$$

Since $\operatorname{Ext}_{R}^{i}(R / P, R)_{\mathfrak{p}}=\operatorname{Ext}_{R_{\mathfrak{p}}}^{i}\left(R_{\mathfrak{p}} / P_{\mathfrak{p}}, R_{\mathfrak{p}}\right)$, (4) shows that

$$
\operatorname{Ext}_{R_{\mathfrak{p}}}^{i}\left(R_{\mathrm{p}} / P_{\mathrm{p}}, R_{\mathrm{p}}\right) \neq 0 \Leftrightarrow i=n-1 \text {. }
$$

By (3) and (5),

$$
\operatorname{pr} \operatorname{dim}_{R_{\mathfrak{p}}}\left(R_{\mathfrak{p}} / P_{\mathfrak{p}}\right)=n-1 .
$$

Since $J\left(R_{\mathfrak{p}}\right)$ has the right AR property [7, 2.1],

$$
\mathrm{rtgl} \operatorname{dim}\left(R_{\mathfrak{p}}\right)=n-1
$$

by (6) and 2.3(i). The theorem follows from (2) and (7), since $k$-dim $\left(R_{\mathfrak{p}}\right)$ coincides with its classical Krull dimension [7, 1.7, 1.8].

3.6. TheOrem. Let $R$ be a ring which is hom. hom. over $C$. Let $P$ be a prime ideal of $R$, of rank $t$. Then

(i) $\operatorname{gr}(P)=G_{C}(P)=t$ and

(ii) if $n=\sup \left\{\operatorname{pr} \operatorname{dim}_{R}(V): V\right.$ a simple $(R / P)$-module $\}$ then

$$
n=\operatorname{gr}(P)+k-\operatorname{dim}(R / P)=G_{C}(P)+k-\operatorname{dim}(R / P) .
$$


Proof. (i) By $2.5, R$ is Macaulay, so $G_{C}(P)=t$ by $[5,4.11]$. By Corollary 3.1, $\operatorname{gr}(P) \geqslant G_{C}(P)$. We claim that

$$
\operatorname{gr}(P) \leqslant G_{C}(P) .
$$

If $P$ is maximal this follows from 2.5. Otherwise, localize at a prime ideal a of $C$ containing $P \cap C$, so that $k-\operatorname{dim}\left(R_{\mathrm{a}} / P_{\mathrm{a}}\right)=1$. Then $G_{C}(P)=G_{C_{\mathrm{a}}}\left(P_{\mathrm{a}}\right)=s$ say, by 3.4 , and

$$
\operatorname{Ext}_{R_{\mathrm{a}}}^{s}\left(R_{\mathrm{a}} / P R_{\mathrm{a}}, R_{\mathrm{q}}\right) \neq 0
$$

by (5) in the proof of 3.5. That is, $\operatorname{gr}(P) \leqslant s$, and (8) is proved.

(ii) Let $\mathrm{m}=r_{C}(V)$, where $V$ is a simple $(R / P)$-module with $\operatorname{pr} \operatorname{dim}_{R}(V)=n$. Then

$$
\begin{aligned}
n & =\mathrm{rtgl} \operatorname{dim}\left(R_{\mathrm{m}}\right), & & \text { by } 2.1 \text { and } 2.3(\mathrm{i}) \\
& =k-\operatorname{dim}\left(R_{\mathrm{m}}\right), & & \text { by } 3.5 \\
& =\operatorname{rank}\left(P_{\mathrm{m}}\right)+k-\operatorname{dim}\left(R_{\mathrm{m}} / P_{\mathrm{m}}\right), & & \text { by }[5,5.1] \\
& =G_{C_{\mathrm{m}}}\left(P_{\mathrm{m}}\right)+k-\operatorname{dim}\left(R_{\mathrm{m}} / P_{\mathrm{m}}\right), & & \text { by }[5,4.11] \\
& \leqslant G_{C}(P)+k-\operatorname{dim}(R / P), & & \text { by } 3.4 \\
& =\operatorname{gr}(P)+k-\operatorname{dim}(R / P), & & \text { by } 3.6(\mathrm{i}) .
\end{aligned}
$$

Now let $\omega=G_{C}(P)+k-\operatorname{dim}(R / P)$, so there exists a maximal ideal $M$ of $R$ with $P \subseteq M$ and $\operatorname{rank}(M)=\omega$. Let $V$ be the simple $R$-module with $V M=0$, so $\operatorname{pr} \operatorname{dim}(V)=\omega$ by 2.5 . Hence, $n \geqslant \omega$, and the proof is complete.

4. The factor by a $C$-sequence. While the factor of a hom. hom. ring $R$ by an ideal $I$ generated by a $C$-sequence need not be hom. hom.-consider $k[X] /\left\langle X^{2}\right\rangle$ - such a ring is $C$-Macaulay and "Gorenstein". Consequently, $R / I$ has a quasi-Frobenius quotient ring, (4.4). This applies in particular when $I=0$, and leads easily to the conclusion that $R$ is semiprime, (4.2).

4.1. We begin by recording a result which was essentially proved in [5], and which is a straightforward analogue of results in the commutative theory [12, Theorems 137 and 141].

Theorem. Let $R$ be a right Noetherian $C$-Macaulay ring, let $x_{1}, \ldots, x_{t}$ be a $C$-sequence on $R$. Put $I=\sum_{i} x_{i} R, \bar{R}=R / I$ and $\bar{C}=[C+I] / C$. Then

(i) $\bar{R}$ is a $\bar{C}$-Macaulay ring.

(ii) $\bar{R}$ has a right Artinian quotient ring.

Proof. (i) Clearly, only the case $t=1$ need be considered. Let $M$ be a maximal ideal of $R$ containing $x_{1}$. Now, setting $\bar{M}=M / I$,

$$
\begin{aligned}
G_{C}^{-}(\bar{M}) & =G_{C}(M)-1 \quad \text { by }[5,4.4] \\
& =\operatorname{rank}(M)-1 \\
& \geqslant 1+\operatorname{rank}(\bar{M})-1=\operatorname{rank}(\bar{M}),
\end{aligned}
$$

where the inequality holds since $x_{1}$ does not lie in any minimal prime of $R$. But $\operatorname{rank}(\bar{M}) \geqslant G_{C}^{-}(\bar{M})$ by 3.3 , so (i) is proved.

(ii) This follows from (i) by [5, 4.13]. 
4.2. COROllaRY. If $R$ is a hom. hom. ring, then $R$ is semiprime.

Proof. By 4.1(ii) $R$ has a right Artinian quotient ring $Q$, which is obtained by localizing at $\bigodot_{Z}(N \cap Z)$, where $N$ is the nilpotent radical of $R$ and $Z$ is the center of $R$. By 3.5, $Q$ is semisimple Artinian.

4.3. To strengthen 4.1 for hom. hom. rings we need a well-known lemma.

Lemma Let $Q$ be a right Artinian ring of finite right injective injective dimension such that every irreducible right $Q$-module occurs in the socle of $Q$. Then $Q$ is quasi-Frobenius.

Proof. Let $Q$ have injective dimension $n$, and suppose $n>0$. Let $V$ be an irreducible right $Q$-module, so there is an exact sequence

$$
0 \rightarrow V \rightarrow Q \rightarrow Q / V \rightarrow 0 \text {. }
$$

This yields the exact sequence

$$
\operatorname{Ext}^{n}(Q, Q) \rightarrow \operatorname{Ext}^{n}(V, Q) \rightarrow \operatorname{Ext}^{n+1}(Q / V, Q) .
$$

Since the outer terms are both zero, $\operatorname{Ext}^{n}(V, Q)=0$. Hence, $\operatorname{Ext}^{n}(B, Q)=0$ for every $Q$-module $B$ of finite length, and so $Q$ has injective dimension at most $(n-1)$, a contradiction. Thus, $n=0$, and $Q$ is quasi-Frobenius [18, Chapter XIV, 3.3].

4.4. THEOREM. Let $R$ be a ring which is hom. hom. over a central subring $C$, and let $x_{1}, \ldots, x_{t}$ be a $C$-sequence on $R$. Set $I=\Sigma_{i} x_{i} R$ and $\bar{R}=R / I$.

(i) The right annihilator primes of $\bar{R}$ are precisely the minimal primes of $\bar{R}$.

(ii) The right quotient ring of $\bar{R}$ exists and is quasi-Frobenius.

(iii) $\bar{R}$ is left Goldie and its left annihilator primes are precisely its minimal primes.

Proof. (i) Since $\bar{R}$ has a right Artinian quotient ring, by 4.1(ii), its right annihilator primes are minimal. Conversely, let $P / I$ be a minimal prime of $\bar{R}$. Then $G_{C}(P)=t$, so $\operatorname{gr}(P)=t$ by $3.6(\mathrm{i})$. That is, $\operatorname{Ext}_{R}^{t}(R / P, R) \neq 0$. By 3.1, $\operatorname{Hom}_{R}(R / P, \bar{R}) \neq 0$. It follows that $P / I$ is contained in a right annihilator prime of $\bar{R}$. The result therefore follows by appealing once more to 4.1(ii).

(ii) The right quotient ring $Q$ of $\bar{R}$ exists by 4.1(ii). Since $R$ has finite right injective dimension, so does $\bar{R}$ by [12, Theorem 205]. Hence $Q$ has finite right injective dimension. By (i) and 4.3, $Q$ is quasi-Frobenius.

(iii) Since $Q$ is quasi-Frobenius, it is left Artinian and left self-injective, so its left socle contains a copy of each of its irreducible left modules [18, Chapter XIV, §3]. Let $N / I$ be the nilpotent radical of $R / I$ and put $\mathfrak{a}=N \cap C$, so $\bigodot_{C}(\mathfrak{a}) \subseteq \bigodot_{R}(I)$ by (ii) and Small's Theorem. Let $\bigodot$ be the image of $\bigodot_{C}(a)$ in $\bar{R}$. The integrality of $\bar{R}$ over $\bar{C}$ implies that $\bar{R}_{\mathcal{C}}$ is the complete right quotient ring $Q$ of $\bar{R}$. Since $\bigodot$ is central, $Q$ is also the left quotient ring of $\bar{R}$. The left hand properties of $Q$ now yield the desired conclusions concerning $\bar{R}$.

5. Direct sums of prime rings. In this section we improve on 4.2 by showing that a hom. hom. ring is a direct sum of prime rings (5.3). 
5.1. Lemma. Let $R$ be a semiprime right Noetherian ring integral over a central subring $C$. Suppose that $R / c R$ has a right Artinian quotient ring for each element $c \in \mathcal{C}_{R}(0) \cap C$. Suppose $R$ is not semisimple Artinian. Then

(i) $R=\bigcap_{\lambda \in \Lambda} R_{\lambda}$, where $k-\operatorname{dim}\left(R_{\lambda}\right)=1$ for all $\lambda \in \Lambda$.

(ii) If $R$ is hom. hom. over $C, \mathrm{rtgl} \operatorname{dim}\left(R_{\lambda}\right)=1$ for all $\lambda \in \Lambda$.

Proof. (i) Let $P_{1}, \ldots, P_{n}$ be the minimal prime ideals of $R$. Let $T$ be a rank 1 prime ideal of $R$. Renumber the $P_{i}$ 's if necessary so that $P_{i} \subseteq T$ for $1 \leqslant i \leqslant k$ and $P_{i} \not T$ for $k+1 \leqslant i \leqslant n$. Let $\bigodot=\bigodot_{C}(T) \cap \cap_{i=k+1}^{n} e_{C}\left(P_{i}\right)$.

Let $I=\{r \in R: r c=0, c \in \mathcal{C}\}$. Thus $I$ is an ideal and $I c=0$ for some $c \in \mathcal{C}$. Since $c \notin P_{i}$ for all $i=1, \ldots, n, I=0$. Hence the localization $R_{e}$ is a subring of the quotient ring of $R$ and $R \subseteq R_{\mathfrak{C}}$. Denote $R_{\mathcal{e}}$ by $R_{T^{*}}$, and note that, its prime ideals having rank at most one, $R_{T^{*}}$, has Krull dimension one.

We claim that

$$
R=\cap\left\{R_{T^{*}}: T \text { rank } 1 \text { prime }\right\} .
$$

Let $q$ be an element in the intersection, and set $X=\{r \in R: r q \in R\}$, a left ideal. Write $q$ as $a c^{-1}$ where $a \in R$ and $c \in \mathcal{C}_{R}(0) \cap C$. By Jategaonkar's Principal Ideal Theorem [8,3.5], the prime ideals minimal over $c R$ have rank one. By definition, $X \cap \bigodot_{R}(T) \neq \varnothing$ for every prime ideal $T$ minimal over $c R$. Hence, $X \cap \bigodot_{R}(N) \neq \varnothing$, where $N / c R$ is the nilpotent radical of $R / c R$. By hypothesis and Small's Theorem, there exists $d \in X \cap \mathcal{C}_{R}(c R)$. Thus $d q \in R$, and so $d c q=c d q \in c R$. Sisce $d \in$ $\bigodot_{R}(c R)$ and $c q \in R, c q \in c R$. Hence, $q \in R$, and (9) is proved.

(ii) Since each $R_{\lambda}$ in (i) is a localization $R_{T^{*}}$ at a semiprime ideal of $C$, this follows from 3.5.

5.2. Lemma. Let $R$ be a semiprime right Goldie ring. Suppose that $R=\bigcap_{\lambda \in \Lambda} R_{\lambda}$, where each $R_{\lambda}$ is a subring of the right quotient ring $Q$ of $R$. If each $R_{\lambda}$ is a direct sum of prime rings, then the same is true of $R$.

Proof. Let $e$ be a central idempotent of $Q$. Since $R_{\lambda}$ is a direct sum of prime rings and has $Q$ as its right quotient ring, $e \in R_{\lambda}$ for every $\lambda \in \Lambda$. Thus $e \in R$.

REMARK. If $R$ is a right order in a right Artinian ring the above result holds more generally with 'direct sum of prime rings' replaced by 'direct sum of prime and right Artinian rings'.

5.3. Commutative Noetherian rings of finite global dimension and semiprime right Noetherian right hereditary rings are both direct sums of prime rings [12, Theorem 168] and [8, 8.4 and 8.23]. The next theorem generalizes the first of these results and partially generalizes the second.

TheOREM. Let $R$ be a hom. hom. ring. Then $R$ is a direct sum of prime hom. hom. rings.

Proof. By 4.2, $R$ is semiprime. It satisfies the hypothesis of 5.1, by 4.1(ii). Hence $R=\bigcap_{\lambda \in \Lambda} R_{\lambda}$, where each $R_{\lambda}$ is a semiprime right hereditary partial quotient ring of $R$. By Levy's theorem [8, 8.4 and 8.23], $R_{\lambda}$ is a direct sum of prime rings. The result follows from 5.2. 
5.4. For every integer $n \geqslant 2$, there exist semiprime Noetherian rings finitely generated over their centers, of global dimension $n$, which do not decompose as a direct sum of prime rings [15]. Thus the hypothesis that $R$ is hom. hom. is crucial in 5.3 .

6. The center of a homologically homogeneous ring. In the light of 5.3 we need consider only the case of a prime hom. hom. ring.

6.1. THEOREM. Let $R$ be a hom. hom. prime ring with $\mathrm{rtgl} \operatorname{dim}(R)=n$. Let $Z=Z(R)$. Then $Z$ is a Krull domain with classical Krull dimension $n$. If $Z$ is Noetherian then $k-\operatorname{dim}(Z)=n$.

Proof. Specialized to the case where $R$ is prime, 5.1 says simply that $R=\bigcap_{\mathrm{p} \in P} R_{\mathrm{p}}$ where $\mathcal{P}$ is the set of rank 1 primes of $Z$. Let $Q$ be the quotient ring of $R$. Then

$$
Z=R \cap Z(Q)=\bigcap_{\mathfrak{p} \in \mathscr{P}} R_{\mathfrak{p}} \cap Z(Q)=\bigcap_{\mathfrak{p} \in \mathscr{P}} Z\left(R_{\mathfrak{p}}\right)=\bigcap_{\mathfrak{p} \in \Phi} Z_{\mathfrak{p}}
$$

If $\mathfrak{p} \in \mathcal{P}, R_{\mathfrak{p}}$ is right hereditary by 3.5 , and so $Z_{\mathfrak{b}}$ is a Krull domain $[1,7.1]$ which is local and of dimension 1. Thus $Z_{\mathfrak{p}}$ is a DVR, and so $Z$ is a Krull domain. Since $R$ is $Z$-Macaulay of dimension $n$, by 2.5 , and $R$ is integral over $Z$, the remaining claims follow from [5, 4.11].

6.2. For the special case of a local ring $R, 6.1$ is $[5,6.6]$.

6.3. Since there exist prime Noetherian rings of arbitrary finite global dimension which are finitely generated over a central Dedekind domain [20], the dimensional aspects of 6.1 clearly require that $R$ be hom. hom. It would seem to be an open question, however, whether the center of a prime Noetherian ring of finite global dimension is necessarily integrally closed (even when the ring is finitely generated over the center).

6.4. In addition to the properties given by 6.1 , the center $Z$ of an $n$-dimensional hom. hom. ring possesses a Cohen-Macaulay module of grade $n$ (namely $R$ ). However, even when $Z$ is Noetherian, it need not itself be Cohen-Macaulay [5, 7.3]. The determination of which commutative domains can appear as the centers of prime right Noetherian hom. hom. rings would appear to be a difficult problem.

7. Polynomial rings and group rings. We discuss here the homological homogeneity of polynomial extensions (7.3), and hom. hom. group rings (7.5).

7.1. It is not hard to use the theory developed in [5, §4] to prove the following result, by mimicking the corresponding argument in the commutative theory [12, Theorem 151]. We leave the details to the reader.

Proposition. Let $R$ be a right Noetherian ring finitely generated as a module over the central subring $C$. If $R$ is $C$-Macaulay then $R[X]$ is $C[X]-M a c a u l a y$. Indeed, if $M$ is a maximal ideal of $R[X]$ and $P=M \cap R$, then

$$
G_{C[X]}(M)=G_{C}(P)+1=\operatorname{rank}(P)+1=\operatorname{rank}(M) .
$$

7.2. The next result is a partial converse to 2.5 . 
Proposition. Let $R$ be a right Noetherian ring integral over the central subring $C$. Suppose that $\mathrm{rt} \operatorname{gl} \operatorname{dim}(R)=n<\infty$ and that $R$ is $C$-Macaulay with $\operatorname{rank}(M)=n$ for all maximal ideals $M$ of $R$. Then $R$ is hom. hom.

Note. The hypothesis on the ranks of the maximal ideals of $R$ is necessary in this result-consider $R=\left(\begin{array}{cc}F & F \\ 0 & F\end{array}\right)$ for a field $F$.

Proof. Let $M$ be a maximal ideal of $R$. Then $G_{C}(M)=\operatorname{rank}(M)=n$, so $\operatorname{Ext}_{R}^{i}(R / M, R)=0$ for $0 \leqslant i \leqslant n-1$ by Corollary 3.1. By hypothesis, $\operatorname{pr} \operatorname{dim}_{R}(R / M) \leqslant n$. If pr $\operatorname{dim}_{R}(R / M)=0, M$ is a right annihilator prime, and so $M$ is minimal by 4.1(ii). In this case $n=0$ and $R$ is semisimple Artinian. Otherwise, $\operatorname{pr} \operatorname{dim}_{R}(R / M)=n>0$. Since this holds for all maximal ideals $M$, the result follows.

7.3. THEOREM. If $R$ is a hom. hom. ring finitely generated as a module over its center, $Z$, then so is $R[X]$.

Proof. Clearly $R[X]$ is integral over $Z[X]$. Let $\operatorname{rtgl} \operatorname{dim}(R)=n<\infty$, so $\mathrm{rtgl} \operatorname{dim}(R[X])=n+1$ by $[16,9.34]$. Let $M$ be a maximal ideal of $R[X]$, let $M \cap Z[X]=\mathrm{m}$, and let $M=M_{1}, \ldots, M_{t}$ be the maximal ideals lying over $\mathrm{m}$. Put $p=\mathfrak{m} \cap Z$. Let $\mathrm{e}=Z \backslash \mathfrak{p}$ and $\mathcal{S}=Z[X] \backslash \mathfrak{m}$. By 2.1

$$
\operatorname{pr} \operatorname{dim}_{R[X]_{s}}\left(R[X]_{\S} / M_{i_{s}}\right)=\operatorname{pr} \operatorname{dim}_{R[X]}\left(R[X] / M_{i}\right)
$$

for $i=1, \ldots, t$. Hence, we need only prove that $R[X]_{\S}$ is hom. hom.

Now $R[X]_{\varrho}=R_{\mathcal{e}}[X]$ is $Z_{\mathcal{e}}[X]$-Macaulay by 3.5, 2.5 and 7.1. Hence $R[X]_{\mathfrak{\varsigma}}$ is $C[X]_{\mathfrak{S}}$-Macaulay by 3.4 . The maximal ideals of $R[X]_{\S}$ are $M_{1_{\S}}, \ldots, M_{t_{\S}}$. For $i=1, \ldots, t$,

$$
\begin{aligned}
\operatorname{rank}\left(M_{i_{5}}\right) & =\operatorname{rank}\left(M_{i}\right)=\operatorname{rank}\left(M_{i} \cap R\right)+1, \quad \text { by } 7.1, \\
& =\operatorname{rank}(\mathfrak{p})+1=\operatorname{rtgldim}\left(R_{\varrho}\right)+1,
\end{aligned}
$$

by 3.5 .

Since $R[X]_{\S}$ is a localization of $R_{\mathrm{e}}[X]$,

$$
\mathrm{rtgl} \operatorname{dim}\left(R[X]_{\S}\right) \leqslant \mathrm{rtgldim}\left(R_{\mathfrak{E}}\right)+1,
$$

by $[16,9.34]$. By $(10),(11)$ and $[5,6.1]$, equality holds in (11). Therefore $R[X]_{\mathfrak{s}}$ is hom. hom. by 7.2 .

7.4. Essentially the same proof can be used to obtain a somewhat stronger statement than 7.3: If $R$ is hom. hom. and finitely generated over $Z$ and $\sigma$ is an automorphism on $R$ of finite order which is the identity on $Z$, then $R[X ; \sigma]$ is hom. hom. We are grateful to R. Resco for pointing out to us that uncertainty over the preservation of integrality under polynomial extension forces us to require, here and in 7.3, that $R$ be a finitely generated $Z$-module.

7.5. Proposition. Let $G$ be a finitely generated group with an abelian normal subgroup $A$ of finite index. Let $S$ be (a) the ring of integers or (b) a field. Suppose in case (a) that $G$ is torsion free and in case (b) that, if char $S=p>0, G$ has no element of order p. Then $S G$ is hom. hom. 
Proof. The ring $S G$ is Noetherian [13, 10.2.8], finitely generated over its center $[13,4.1 .10]$, and has global dimension equal to $h(G)+1$ [resp. $h(G)]$ is case (a) [resp. case (b)], where $h(G)$ denotes the Hirsch number of $G,[16,9.3]$ and $[19,9.2]$. Clearly, we may assume that $A$ is torsion free. Let $n$ be the global dimension of $S G$, and let $V$ be an irreducible $S G$-module. By [13, 7.2.16] $V$ has finite length as an $S A$-module, and so $\operatorname{pr}^{\operatorname{dim}_{S A}}(V)=n$ by the commutative theory (or by 2.5 ). Since $S G$ is $S A$-free, pr $\operatorname{dim}_{S G}(V) \geqslant n$. Hence pr $\operatorname{dim}_{S G}(V)=n$ and $S G$ is hom. hom.

\section{Concluding remarks.}

8.1. As mentioned in $\S 1$, the results of this paper generalize those of [5] on a local ring $R$ of finite right global dimension integral over its center. There is one important point where the theory fails to extend, however: If $R$ is local and hom. hom., then it is a maximal order [10]. For arbitrary hom. hom. rings, this is false - consider for example the ring $\left[\begin{array}{ll}\mathbf{Z} & \mathbf{Z} \\ 2 \mathbf{Z} & \mathbf{Z}\end{array}\right]$. In fact it is not hard to deduce from 5.1 and [6, Corollary 3.8] that a Noetherian hom. hom. ring is a direct sum of maximal orders if and only if all its rank one primes are localizable.

8.2. The results of this paper have all been obtained for a right Noetherian hom. hom. ring. But we have no example of such a ring which is not left Noetherian - we leave the existence of such rings as an open question. A hom.hom. ring satisfying a P.I. is Noetherian by 4.2 and $[17,5.1 .7]$. In dimension one, no one-sided examples can exist, by 4.4(ii).

8.3. The key properties of hom.hom. rings are that they have finite global dimension and that the projective dimensions of "closely related" irreducible modules are the same. Consideration of other examples, notably group rings and enveloping algebras, leads us to suspect that the integrality hypothesis (while crucial to our proofs), may be much less significant. For example, the equality of the Krull and global dimensions, and the decomposition as a direct sum of prime rings, may be valid in much more general circumstances than those treated here. We state without proof the following sample result, which can be proved quite easily using a result of Small [11, Proposition 4] and the techniques of this paper:

Let $R$ be a prime affine P.I. ring of finite right global dimension $n$ such that for all irreducible $R$-modules $V$ and $W$ with $r_{Z}(V)=r_{Z}(W), \operatorname{pr} \operatorname{dim}(V)=\operatorname{pr} \operatorname{dim}(W)$. Then $k-\operatorname{dim}(R)=n$.

Note that not all prime Noetherian affine P.I. rings satisfy this homogeneity hypothesis, nor the conclusion-see [14, 1.7 and 1.8].

\section{REFERENCES}

1. G. M. Bergman and P. M. Cohn, The centres of 2-firs and hereditary rings, Proc. London Math. Soc. 23 (1971), 83-98.

2. J. Bernstein, I. Gelfand and S. Gelfand, Structure locale de la catégorie des modules de Harish-Chandra, C. R. Acad. Sci. Paris 286 (1978), 495-497.

3. S. M. Bhatwadekar, On the global dimension of some filtered algebras, J. London Math. Soc. (2) 13 (1976), 239-248.

4. K. A. Brown, C. R. Hajarnavis and A. B. MacEacharn, Noetherian rings of finite global dimension, Proc. London Math. Soc. 44 (1982), 349-371.

5. __ Rings of finite global dimension integral over their centers, Comm. Algebra 11 (1983), 67-93. 
6. M. Chamarie, Localisations dans les ordres maximaux, Comm. Algebra 2 (1974), 279-293.

7. M. Chamarie and A. Hudry, Anneaux noethériens à droite entiers sur un sous-anneau de leur centre, Comm. Algebra 6 (1978), 203-222.

8. A. W. Chatters and C. R. Hajarnavis, Rings with chain conditions, Pitman, London, 1980.

9. R. Gordon and J. C. Robson, Krull dimension, Mem. Amer. Math. Soc., no. 133, 1973.

10. A. Gray, A class of maximal orders integral over their centres, Glasgow Math. J. (to appear).

11. S. Jøndrup, Homological dimensions of some P. I. rings, Comm. Algebra 8 (1980), 685-696.

12. I. Kaplansky, Commutative rings, Allyn and Bacon, Boston, Mass., 1970.

13. D. S. Passman, The algebraic structure of group rings, Interscience, New York, 1977.

14. R. Resco, L. W. Small and J. T. Stafford, Krull and global dimensions of semiprime Noetherian PI-rings, Trans. Amer. Math. Soc. 274 (1982), 285-295.

15. J. C. Robson, Some constructions of rings of finite global dimension (to appear).

16. J. J. Rotman, An introduction to homological algebra, Academic Press, New York, 1979.

17. L. H. Rowen, Polynomial identities in ring theory, Academic Press, New York, 1980.

18. B. Stenström, Rings of quotients, Springer-Verlag, Berlin and New York, 1975.

19. R. G. Swan, Groups of cohomological dimension one, J. Algebra 12 (1969), 585-610.

20. R. B. Tarsy, Global dimension of orders, Trans. Amer. Math. Soc. 151 (1970), 335-340.

21. V. W. Vasconcelos, On quasi-local regular algebras, Symposia Math., Vol. XI, Academic Press, London, 1973.

Department of Mathematics, University of Glasgow, G12 80W Glasgow, Scotland

Mathematics Institute, University of WaRwick, Coventry CV4 7AL, ENGland (Current address of C. R. Hajarnavis)

Current address (K. A. Brown): Department of Mathematics, University of Washington, Seattle, Washington 98195 